\title{
eHealth im klinischen Alltag, mit Blick aus der Neuen Welt
}

\section{Yvonne Gilli}

Dr. med., Mitglied des FMH-Zentralvorstandes, Departementsverantwortliche Digitalisierung / eHealth

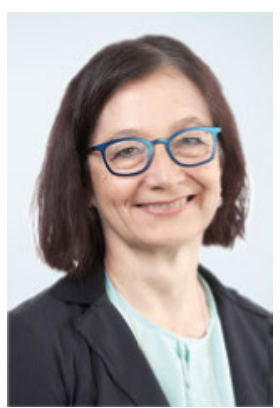

Nur wenige Tage nach der Wahl des 45. Präsidenten von Amerika begann in Chicago das 40. AMIA-Symposium¹. AMIA steht für "American Medical Informatics Association». Das Symposium bietet eine unabhängige wissenschaftliche Plattform für den internationalen und interprofessionellen Erfahrungsaustausch zu Medizininformatik, Computertechnologie und Gesundheitsversorgung.

Mit dem elektronischen Patientendossier will die Schweiz einen Impuls setzen für die Digitalisierung im Gesundheitswesen. Die Politik hofft gar auf eine Effizienzsteigerung mit Kostenreduktion. Dieses Ziel ist unter den gegebenen Rahmenbedingungen kaum zu erreichen: Zu klein sind die finanziellen Anreize, um die klinikeigenen Informatiksysteme und individuellen Praxislösungen zukunftsfähig zusammenzuführen. Zu gross ist der Wissens- und Ausbildungsrückstand im Bereich der Medizininformatik, heute bereits ein Trend- und Mangelberuf in den USA.

\section{Den Erfahrungsvorsprung anderer Länder} gilt es bei der Ausgestaltung des elektronischen Patientendossiers zu nutzen.

Auf Initiative der FMH nahm eine Delegation der Interprofessionellen Arbeitsgruppe elektronisches Patientendossier (IPAG EPD) am AMIA-Kongress teil. Dominik Aronsky ${ }^{2}$ ermöglichte uns zwei Besuche in führenden Kliniken, dem Vanderbilt University Medical Center in Nashville und bei Partners HealthCare in Boston.

1 www.amia.org

2 Prof. Dominik Aronsky, MD, PhD, FAC, studierte Medizin an der Universität Bern und Medizininformatik an der University of Utah, Vanderbilt University, Nashville. Mandatiert von der FMH als Experte der IPAG EPD

3 A Terminology Framework for Nursing Documentation Redesign, Deborah Ariosto, $\mathrm{PhD}$ MSN, RN. Vanderbilt University Medical Center, Nashville, TN. AMIA Symposium 2016.
Praxisbezogene Impulsreferate und der Besuch einer grossen Notfallstation sensibilisierten uns für das Potential, das in der Schweiz noch brach liegt. Meine wichtigste Take-Home Message ist einfach: Wir dürfen profitieren vom Erfahrungsvorsprung in den USA und einigen europäischen Ländern, sofern wir ihre Erkenntnisse in unsere Prozesse integrieren. den Austausch von Patientendaten betrifft, sind wir mit den gleichen Herausforderungen konfrontiert. Software- und Hardwarelösungen müssen benutzerfreundlich sein und einen Mehrwert generieren. «Der Was die elektronische Erfassung, Bewirtschaftung und
Informatiker kennt den Wert von Standards für die Verwendung von Daten, Interoperabilität, Systemstabilität und Werkzeuge für die Entscheidungsunterstützung. Der Kliniker kennt seine eigenen Praxisabläufe und Bedürfnisse. In der Zusammenarbeit erreichen beide ihre Ziele. ${ }^{3}$ Dies ist die Schlussfolgerung eines Forschungsprojekts am Vanderbilt Hospital: Über fünfzig Pflegeverantwortliche auf Akutstationen inklusiv Pädiatrie stellten sich erfolgreich der Herausforderung, das elektronische Dokumentationssystem so zu reorganisieren, dass die administrative Belastung sinkt. Wichtige Probleme erkannten sie in redundanter oder unnötiger Dokumentation und in den Kommunikationsabläufen innerhalb des Betreuungsteams. Mit Hilfe von interdepartementalen Arbeitsgruppen konnten sie die Dokumentationszeit pro Pflegeperson um eine halbe Stunde pro Tag senken und das Dokumentationsvolumen pro Patient um ein Drittel reduzieren. Ich gehe davon aus, dass für Ärztinnen und Ärzte das gleiche Potential brach liegt und für mehr Zufriedenheit und Zeit für direkten Patientenkontakt zu nutzen wäre.

Dies ist ein einfaches Beispiel der Herausforderungen im klinischen Alltag. Die Referate des AMIA-Symposiums umfassen ein Volumen von über zweitausend Seiten und berühren praktisch jedes Thema, das auch uns unter den Nägeln brennt, von der eMedikation bis zu Big Data. Für exzellente Lösungen «folgt» die Informatik den komplexen klinischen Abläufen und nicht um-

\section{Für exzellente Lösungen folgt die Informatik} den komplexen klinischen Abläufen und nicht umgekehrt.

gekehrt. Die Brücke zwischen den beiden Disziplinen schlagen unter anderen die Medizininformatikerinnen. Die Förderung von Ausbildung, Forschung und Anwendung ist möglich mit geeigneten politischen Rahmenbedingungen. Sie kosten etwas mehr, als die Schweiz bis jetzt noch zu investieren bereit ist in eine Zukunft, die unsere Gesundheitsversorgung grundlegend verändern wird. 